\title{
Interacting Entropy-Corrected Holographic Dark Energy and IR Cut-Off Length
}

\author{
J. Sadeghi ${ }^{a *}$ B. Pourhassan ${ }^{b \dagger}$ and Z. Abbaspour Moghaddam ${ }^{a \ddagger}$ \\ a Faculty of Basic Sciences, Departments of Physics, Mazandaran University, \\ P.O.Box 47416-95447, Babolsar, Iran \\ ${ }^{b}$ Department of Physics, I.H.U., Tehran, Iran
}

October 30, 2018

\begin{abstract}
In this paper we consider holographic dark energy model with corrected holographic energy density and show that this model may be equivalent to the modified Chaplygin gas model. Then we obtain relation between entropy corrected holographic dark energy model and scalar field models. We do these works by using choices of IR cut-off length proportional to the Hubble radius, the event horizon radius, the Ricci length, and the Granda-Oliveros length.
\end{abstract}

Keywords: Holographic Dark Energy; Modified Chaplygin Gas; Scalar Field.

\section{Introduction}

Astrophysical observations of type Ia supernovae [1, 2], the large-scale structure [3], the cosmic microwave back grand radiation [4] suggest accelerated expansion of universe which may be described by the dark energy $[5,6]$. The simplest model to describe dark energy is the cosmological constant, but this model suffers from the cosmic coincidence problems and the fine-tuning. Another suggested models consist of k-essence [7, 8], tachyon [9], quintom [10], quintessence [11] and phantom [12]. Also, there is an interesting model based on equation of state of Chaplygin gas and its generalizations [13-20]. On the other hand, one can investigate the nature of dark energy based on some principles of quantum gravity, which is yield to holographic dark energy (HDE) model [21-29].

In the recent work [30], interacting HDE based on scalar field models has been studied and

\footnotetext{
*Email: pouriya@ipm.ir

†Email: b.pourhassan@umz.ac.ir

${ }^{\ddagger}$ Email: kmabbaspour@yahoo.com
} 
found that interacting HDE model can be considered as the modified Chaplygin gas model. In that case it is possible to study interacting entropy-corrected holographic dark energy models $[31,32,33]$. So, in the Refs. [31, 32] a correspondence between the tachyon, K-essence and dilaton scalar field models established with the interacting entropy-corrected holographic dark energy model in non-flat FRW universe, and dynamics of these scalar fields according to the evolutionary behavior of the interacting entropy-corrected holographic dark energy model has been studied. Then Ref. [33] extended previous works to the case of variable gravitational constant and obtained the equation of state and the deceleration parameters of the interacting viscous entropy-corrected holographic dark energy model. Also, the potential and the dynamics of the quintessence, tachyon, K-essence and dilaton scalar field models according to the evolutionary behavior of the interacting viscous entropy-corrected holographic dark energy model with time-varying $G$ reconstructed.

Now, in this paper we would like to obtain relation between interacting entropy-corrected HDE model and modified Chaplygin gas, also with scalar fields by using different choices of IR cut-off length.

\section{Entropy-corrected holographic dark energy}

It is found that the HDE density may be obtained from the entropy bound [34]. In the framework of black hole thermodynamics [35-46], there is a maximum entropy which is called the Bekenstein-Hawking entropy bound $\left(S_{B H}=A / 4 G\right)$, which is yield to the following relation for the HDE density [33],

$$
\rho_{d} \leq 3 c^{2} M_{p}^{2} L^{-2}
$$

where $c$ is a numerical constant (however it may be considered as a non-constant parameter $[47,48]), M$ is the reduce Planck mass, and $L$ shows the IR cut-off length. In the restriction (1), equality is given if the holographic bound is saturated. In order to consider the quantum effects the Bekenstein-Hawking entropy may be corrected as the following [49, 50, 51],

$$
S=\frac{A}{4 G}+\widetilde{\gamma} \ln \left(\frac{A}{4 G}\right)+\widetilde{\beta}
$$

where $\widetilde{\gamma}$ and $\widetilde{\beta}$ are undetermined constants. By using the relation (2) the entropy-corrected HDE proposed in the following form [52],

$$
\rho_{d}=3 c^{2} L^{-2}+\gamma L^{-4} \ln L^{2}+\beta L^{-4},
$$

where $\gamma$ and $\beta$ are dimensionless constants. In this paper we use several choice of IR cut-off length such as $L=H^{-1}$ (the Hubble radius), $L=R_{E}$ (the radius of the event horizon), $L=\left(H^{2}+\dot{H}\right)^{\frac{-1}{2}}$ (the Ricci length), and $L=\left(\alpha H^{2}+\beta \dot{H}\right)^{\frac{-1}{2}}$ (the Granda-Oliveros length). 


\section{Entropy corrected HDE model and modified Chap- lygin gas}

As we know the FRW universe is described by the following line element,

$$
d s^{2}=-d t^{2}+a^{2}(t)\left(\frac{d r^{2}}{1-k r^{2}}+r^{2} d \Omega^{2}\right)
$$

where $k=0,+1,-1$ are corresponding to flat, closed and open universe. The first Friedman equation is given by,

$$
H^{2}+\frac{k}{a^{2}}=\frac{8 \pi G}{3}\left(\rho_{d}+\rho_{m}\right),
$$

where $\rho_{d}$ is the energy density for the entropy corrected single HDE model and $\rho_{m}$ is the energy density of dark matter. Also the conservation energy equation separated as the following,

$$
\dot{\rho_{m}}+3 H \rho_{m}=Q
$$

and

$$
\dot{\rho_{d}}+3 H \rho_{d}(1+\omega)=-Q,
$$

where equation of state $\omega=\frac{P_{d}}{\rho_{d}}$ is used. Also, $Q$ is the interacting term of the form,

$$
Q=3 H \lambda \rho_{d},
$$

where $\lambda$ is a dimensionless positive parameter. Therefore we can rewrite equations (6) and (7) as the following,

$$
\dot{\rho_{m}}+3 H \rho_{m}\left(1-\frac{\lambda}{u}\right)=0,
$$

and

$$
\dot{\rho}_{d}+3 H \rho_{d}(1+\lambda+\omega)=0,
$$

where $u=\frac{\rho_{m}}{\rho_{d}}$. Adding both equations (6) and (7) gives,

$$
\dot{\rho}_{t}+3 H\left(1+\omega_{t}\right) \rho_{t}=0,
$$

where $\rho_{t}=\rho_{d}+\rho_{m}$, and

$$
\omega_{t}=\frac{P_{d}}{\rho_{d}+\rho_{m}}=\frac{\omega \Omega_{d}}{1+\Omega_{k}},
$$

where the fractional energy densities are defined as,

$$
\begin{gathered}
\Omega_{k}=\frac{k}{a^{2} H^{2}}, \\
\Omega_{d}=\frac{\rho_{d}}{3 M_{p}^{2} H^{2}}, \\
\Omega_{m}=\frac{\rho_{m}}{3 M_{p}^{2} H^{2}},
\end{gathered}
$$


which yield to the following relation,

$$
1+\Omega_{k}=\Omega_{d}+\Omega_{m}
$$

In the following we use unitary transformation $M_{p}^{2}=8 \pi G=1$.

Now we investigate relation between entropy corrected HDE model and modified Chaplygin gas with the following equation of state,

$$
P=A \rho-\frac{B}{\rho^{n}}
$$

with $0<A \leq 1, B>0$, and $n>0$. We would like to examine the equation (3) for the following cases.

\subsection{Hubble radius as IR cut-off}

In that case we use,

$$
L=H^{-1}
$$

in the equation (3) which gives,

$$
\rho_{d}=3 c^{2} H^{-2}+\gamma H^{-4} \ln H^{2}+\beta H^{-4} .
$$

Using the equations (5), (10) and (19) give the following equation of state parameter,

$$
\omega=-1+\frac{u C-\lambda}{1-C}
$$

where $C \equiv c^{2}-\frac{H^{2}}{3}(4 \gamma \ln H+\gamma+2 \beta)$. So, one can obtain,

$$
\omega_{t}=\left(-1+\frac{u C-\lambda}{1-C}\right) \Omega_{d}
$$

On the other hand, the MCG model has the following equation of state parameter,

$$
\omega_{C G}=A-\frac{B}{\rho^{n+1}}
$$

where we used the equation (17) and $\omega_{C G}=P / \rho$. By using modified Chaplygin gas equation of state in the energy conservation relation one can obtain scale factor-dependent energy density as the following,

$$
\rho^{n+1}=\frac{1}{A+1}\left(B+\frac{\delta}{a^{\mu}}\right)
$$

where $\mu=3(A+1)(n+1)$ and $\delta$ is the integration constant. Substituting (23) in (22) and assuming $\omega_{C G}=\omega_{t}$ give,

$$
A-\frac{B(A+1)}{B+\frac{\delta}{a^{\mu}}}=\left(-1+\frac{u C-\lambda}{1-C}\right) \Omega_{d} .
$$


Therefore we obtain,

$$
\sqrt{\frac{\rho_{d}(0)}{3}}\left(t-t_{0}\right)=\int \frac{a^{\frac{1}{2}(3 C(u-\lambda)-2)}}{\sqrt{1-\frac{u C-\lambda}{1-C}}} \sqrt{\frac{B-\frac{\delta A}{a^{\mu}}}{B+\frac{\delta}{a^{\mu}}}} d a
$$

Thus, the entropy-corrected HDE model for Hubble radius as IR cut-off can be considered as modified Chaplygin gas model.

\subsection{Event horizon radius as IR cut-off}

In that case we use,

$$
L=R_{E}
$$

in the equation (3). For the event horizon, the radius is chosen in the form of $L=\operatorname{ar}(t)$ where the function $r(t)$ obtained from the following relation,

$$
\int \frac{d r}{\sqrt{1-k r^{2}}}=\int \frac{d t}{a}=\frac{R_{E}}{a}
$$

with $r(t)=\frac{1}{\sqrt{k}} \sin y$. Therefore we can obtain,

$$
\omega=-1-\lambda-\frac{1}{\sqrt{3}} \mathcal{A}+\sqrt{\frac{\Omega_{d}}{3}} D,
$$

where

$$
\begin{aligned}
\mathcal{A} & \equiv c^{2}+\frac{L^{-2}}{3}\left(\gamma \ln L^{2}+\beta\right) D, \\
D & \equiv \frac{-6 c^{2}-8 \gamma\left(L^{-2} \ln L-1\right)-4 \beta L^{-2}}{\left(3 c^{2}+L^{-2}(\gamma \ln L+\beta)\right)^{\frac{3}{2}}} .
\end{aligned}
$$

Now from the equation (12) we have,

$$
-\frac{B-\frac{\delta A}{a^{\mu}}}{B+\frac{\delta}{a^{\mu}}}=\left(-1-\lambda-\frac{1}{\sqrt{3}} \mathcal{A}+\sqrt{\frac{\Omega_{d}}{3}} D\right) \Omega_{d},
$$

which may be rewritten as the following equation,

$$
\theta x^{3}-\tau x^{2}+\xi=0
$$

where $\theta=\frac{D}{\sqrt{3}}, \tau=\left(1+\lambda+\frac{\mathcal{A}}{\sqrt{3}}\right), \xi=\frac{B-\frac{\delta A}{a^{\mu}}}{B+\frac{\delta A}{a^{\mu}}}$, and $x=\sqrt{\Omega_{d}}$. Hence, we obtain,

$$
\begin{aligned}
\sqrt{\Omega_{d}} & =\frac{\sqrt[3]{-108 \xi \theta^{2}+8 \tau^{3}+12 \sqrt{-3 \xi\left(-27 \xi \theta^{2}+4 \tau^{3}\right)} \theta}}{6 \theta} \\
& +\frac{2 \tau^{2}}{3 \theta \sqrt[3]{-108 \xi \theta^{2}+8 \tau^{3}+12 \sqrt{-3 \xi\left(-27 \xi \theta^{2}+4 \tau^{3}\right)}}}+\frac{\tau}{3 \theta} .
\end{aligned}
$$

From the Fig. 1 we can see that the parameter $\gamma$ increased the $\Omega_{d}$ while the parameter $\beta$ decreased one. 


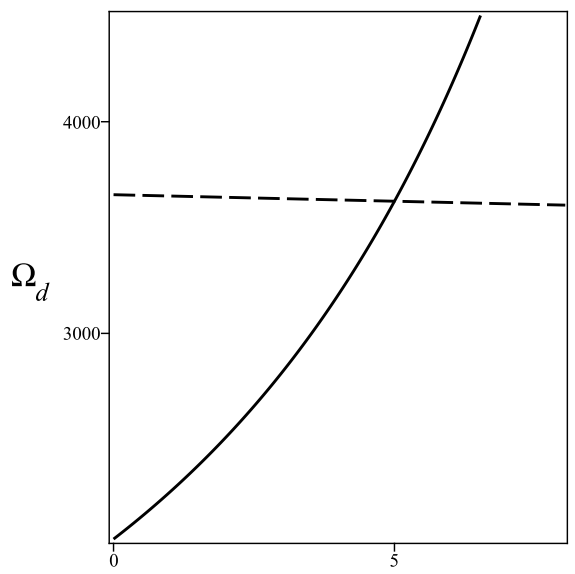

Figure 1: Typical behavior of $\Omega_{d}$ with $\gamma$ (solid line) and $\beta$ (dashed line).

\subsection{Ricci length scale as IR cut-off}

In that case we use,

$$
L=\left(2 H^{2}+\dot{H}\right)^{\frac{-1}{2}}
$$

in the equation (3), and after some calculations obtain,

$$
\Omega_{d}=\frac{7 B-\delta\left(\frac{3 A-4}{a^{\mu}}\right)}{\left(B+\frac{\delta}{a^{\mu}}\right)(2 \eta+3(u+1))},
$$

where, $\eta=c^{2}+\frac{1}{3 L^{2}}(\gamma \ln L+\beta)$. We find that $\Omega_{d}$ is always positive (see Fig. 2), which is agree with the Ref. [30]

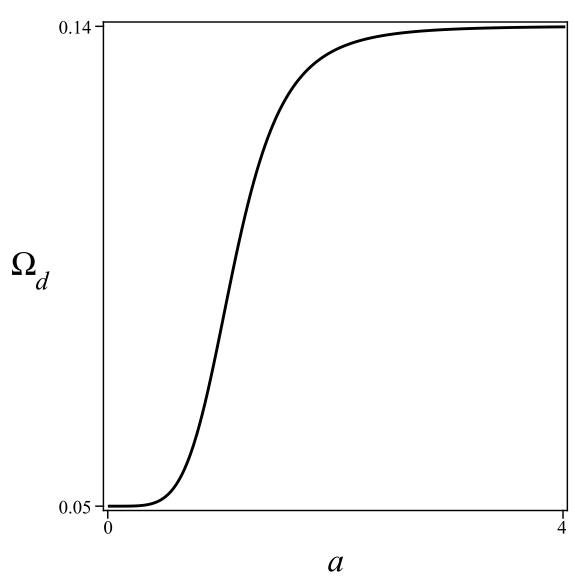

Figure 2: Typical behavior of $\Omega_{d}$ in terms of scale factor. 


\subsection{Granda-oliveros length scale as IR cut-off length}

In that case we use $[53,54]$,

$$
L=\left(\alpha H^{2}+\beta \dot{H}\right)^{\frac{-1}{2}},
$$

in the equation (3), and after some calculations and compare with modified Chaplygin gas obtain,

$$
\Omega_{d}=\frac{B(3 \beta+2 \alpha)+\delta\left(\frac{-3 \beta A+2 \alpha}{a^{\mu}}\right)}{3 \beta\left(B+\frac{\delta}{a^{\mu}}\right)\left(\frac{2}{3 \beta}+(u+1)\right)} .
$$

\section{Entropy-corrected HDE model and scalar field mod- els}

In this section we consider three models of scalar field and investigate relation with entropy corrected HDE model.

\subsection{K-essence}

The general form of the action for K-essence scalar field written as the following,

$$
S=\int d^{4} X \sqrt{-g} P(\Phi, X)
$$

where $P(\Phi, X)$ is pressure density and $X=\frac{-\dot{\Phi}^{2}}{2}$. The energy density and pressure represented as the following,

$$
\rho(\Phi, X)=f(\Phi)\left(-X+3 X^{2}\right),
$$

and

$$
P(\Phi, X)=f(\Phi)\left(-X+X^{2}\right),
$$

where $f(\Phi)$ represents the K-essence potential, and in comparison with entropy corrected HDE model we obtain,

$$
X=\frac{1-\omega}{1-3 \omega} .
$$

\subsubsection{Event horizon radius as IR cut-off}

In that case by using the relation (26) we can obtain,

$$
X=\frac{2+\lambda+\frac{\mathcal{A}}{\sqrt{3}}-\sqrt{\frac{\Omega_{d}}{3}} D}{4+3 \lambda+\sqrt{3} \mathcal{A}-\sqrt{3 \Omega_{d}} D},
$$

which yields to the following expression,

$$
f(\Phi)=\frac{\rho_{d}}{3 X^{2}-X}=\frac{3 H^{2} \Omega_{d}\left(4+3 \lambda+\sqrt{3} \mathcal{A}-\sqrt{3 \Omega_{d}} D\right)}{2\left(2+\lambda+\frac{\mathcal{A}}{\sqrt{3}}-\sqrt{\frac{\Omega_{d}}{3}} D\right)} .
$$


In order to have a real K-essence scalar field we should have the following restriction,

$$
\frac{1}{3}\left(\frac{4+3 \lambda+\sqrt{3} \mathcal{A}}{D}\right)^{2}<\Omega_{d}<3\left(\frac{2+\lambda+\frac{\mathcal{A}}{\sqrt{3}}}{D}\right)^{2} .
$$

\subsubsection{Ricci length scale as IR cut off}

Now, by using the relation (33) we obtain,

$$
X=\frac{\frac{2}{3 \Omega_{d}}\left[\frac{\Omega_{d}}{\eta}-2\right]+(u+2)}{\frac{2}{\Omega_{d}}\left[\frac{\Omega_{d}}{\eta}-2\right]+(3 u+4)} .
$$

Therefore, we obtain,

$$
f(\Phi)=\frac{3 H^{2} \Omega_{d}\left[\frac{2}{\Omega_{d}}\left(\frac{\Omega_{d}}{\eta}-2\right)+(3 u+4)\right]^{2}}{2\left(2+\frac{2}{3 \Omega_{d}}\left(\frac{\Omega_{d}}{\eta}-2\right)+u\right)} .
$$

In order to have a real K-essence scalar field we should have the following restriction,

$$
\frac{4}{\frac{2}{\eta}+3 u+6}<\Omega_{d}<\frac{4}{\frac{2}{\eta}+3 u+4} .
$$

From the equation,

$$
\dot{\Phi}^{2}=-2 X
$$

we can write the explicit form of the K-essence scalar field as,

$$
\Phi=\sqrt{2} \int \sqrt{\frac{\frac{2}{3 \Omega_{d}}\left(\frac{\Omega_{d}}{\eta}-2\right)+(u+2)}{\frac{-2}{\Omega_{d}}\left(\frac{\Omega_{d}}{\eta}-2\right)-(3 u+4)}} d t .
$$

\subsection{Tachyon field}

The effective lagrangian for the tachyon field is described by,

$$
L=-V(\Phi) \sqrt{1-g^{\mu \nu} \partial \mu^{\Phi} \partial \nu^{\Phi}}
$$

The energy density and pressure for the tachyon field are given by the following expressions,

$$
\rho=\frac{V(\Phi)}{\sqrt{1-\dot{\Phi}^{2}}}
$$

and,

$$
P=-V(\Phi) \sqrt{1-\dot{\Phi}^{2}}
$$

In order to find relation between tachyon field and entropy corrected HDE model we consider cases of event horizon radius and Ricci length scale as IR cut-off. 


\subsubsection{Event horizon radius as IR cut-off}

In that case by using the relation (26) we can obtain,

$$
\Phi=\int \frac{\sqrt{-\lambda-\frac{\mathcal{A}}{\sqrt{3}}+\sqrt{\frac{\Omega_{d}}{3}} D}}{H \Omega_{d}\left[-3+\left(1-\Omega_{d}\right)\left(\sqrt{3} \mathcal{A}-\sqrt{3} \Omega_{d} D\right)-3(1+\lambda) \Omega_{d}\right]} d \Omega_{d}+\Phi_{0},
$$

and,

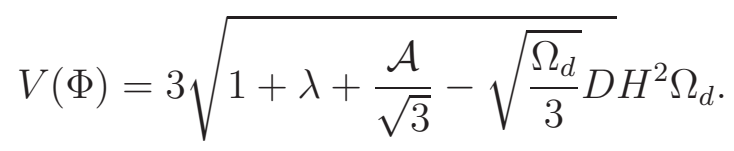

\subsubsection{Ricci length scale as IR cut off}

Now, by using the relation (33) we obtain,

$$
\Phi=\int \sqrt{2-\frac{2}{3 \Omega_{d}}\left(\frac{\Omega_{d}}{\eta}-2\right)-u d t}+\Phi_{0}
$$

and,

$$
V(\Phi)=3 \sqrt{\frac{2}{3 \Omega_{d}}\left(\frac{\Omega_{d}}{\eta}-2\right)+(u-1)} H^{2} \Omega_{d} .
$$

Relation (55) tells that the correction parameters which involves in $\eta$ decreased potential $V(\Phi)$.

\subsection{Quintessence}

The energy density and pressure for the quintessence are given by,

$$
\rho_{\Phi}=\frac{1}{2} \dot{\Phi}^{2}+V(\Phi)
$$

and

$$
P_{\Phi}=\frac{1}{2} \dot{\Phi}^{2}-V(\Phi)
$$

where $\Phi$ is a quintessence field with potential $V(\Phi)$. Here, we assumed $\rho_{\Phi}=\rho_{d}$ and $P \Phi=P_{d}$. Therefore, one can be obtain,

$$
\Phi=\int \frac{\sqrt{\left(1+\omega_{d}\right)} \sqrt{\left(3 \Omega_{d}\right)}}{\Omega_{d}^{\prime}} d \Omega_{d}
$$

and,

$$
V(\Phi)=\frac{3}{2}\left(1-\omega_{d}\right) H^{2} \Omega_{d}
$$

where,

$$
\Omega_{d}^{\prime}=\Omega_{d}\left[-3+\left(1-\Omega_{d}\right)\left(\sqrt{3} \mathcal{A}-\sqrt{3 \Omega_{d}} D\right)-3(1+\lambda) \Omega_{d}\right]
$$




\subsubsection{Event horizon radius as IR cut-off}

In this case one obtain,

$$
\Phi=2 \int \frac{\sqrt{\frac{1}{\sqrt{3}}(x D-\mathcal{A})-\lambda}}{\left[-\sqrt{3}+\left(1-x^{2}\right)(\mathcal{A}-x D)-\sqrt{3}(1+\lambda) x^{2}\right]} d x+\Phi_{0}
$$

where $x \equiv \sqrt{\Omega_{d}}$ defined as before, and,

$$
V(\Phi)=\frac{3}{2} H^{2}\left(2+\lambda+\frac{\mathcal{A}}{\sqrt{3}}-\sqrt{\frac{\Omega_{d}}{3}} D\right) \Omega_{d} .
$$

Thus, $\Phi$ and $V(\Phi)$ are obtain as a function of the density parameter $\Omega_{d}$.

\subsubsection{Ricci length scale as IR cut-off}

By using the relation (33) we obtain,

$$
\Phi=\sqrt{4-\left[\frac{2}{\eta}-3 u\right]\left[\frac{7 B a^{\mu}+\delta(3 A+4)}{\left(B a^{\mu}+\delta\right)(2 \eta+3(u+1))}\right]} \frac{d a}{a}+\Phi_{0},
$$

and,

$$
V(\Phi)=\left(3+\frac{1}{\eta}+\frac{3}{2} u\right)\left(\frac{7 B a^{\mu}-\delta(3 A-4)}{\left(B a^{\mu}+\delta\right)(2 \eta+3(u+1))}\right) .
$$

It is clear that the entropy correction parameters decreased value of potential.

\section{Conclusion}

In this paper, entropy-corrected holographic dark energy model considered for various IR cut-off such as Hubble radius, event horizon radius, Ricci length scale, and Granda-Oliveros length scale. Indeed we extended the Ref. [30] to the case of entropy corrected with parameters $\gamma$ and $\beta$. We investigated relation of entropy-corrected holographic dark energy model with modified Chaplygin gas and scalar field models. We found that correction parameters may be increased or decreased dark energy density. Indeed, we found that the first correction term (second terms of right hand side of the equation (3)) increased dark energy density, while the second correction includes parameter $\beta$ decreased dark energy density. Therefore appropriate choices of $\gamma$ and $\beta$ may be canceled each other. Also we found that the correction parameters reduced potential of scalar fields.

Here, there are some interesting problems such as adding shear and bulk viscosity to system and repeat discussion of this paper. Also one can use explicit expression of scale factor in terms of time and discuss time-dependent solutions. 


\section{References}

[1] A.G. Riess et al., Astron. J. 116 (1998) 1009

[2] S. Perlmutter et al., Astrophys. J. 517 (1999) 565

[3] M. Tegmark et al., Phys. Rev. D69 (2004) 103501

[4] D. N. Spergel et al., Astrophys. J. Suppl. Ser.148 (2003) 175

[5] A.K. Yadav, F. Rahaman, S. Ray, International Journal of Theoretical Physics 50 (2011) 871

[6] H. Saadat, International Journal of Theoretical Physics 50 (2011) 140

[7] C. Armendariz-Picon, V. F. Mukhanov, and P. J. Steinhardt, Phys. Rev. Lett. 85 (2000) 4438

[8] C. Armendariz-Picon, V. F. Mukhanov, and P. J. Steinhardt, Phys. Rev. D 63 (2001) 103510

[9] M. R. Setare, J. Sadeghi, A.R. Amani, Phys. Lett. B673 (2009) 241

[10] B. Feng, X.L. Wang, and X.M. Zhang, Phys. Lett. B 607 (2005) 35

[11] C. Wetterich, Nucl. Phys. B 302 (1988) 668

[12] R.R. Caldwell, Phys. Lett. B 545 (2002) 23

[13] H. Saadat and B. Pourhassan "Viscous Varying Generalized Chaplygin Gas with Cosmological Constant and Space Curvature", International Journal of Theoretical Physics, (2013) DOI10.1007/s10773-013-1676-2

[14] B. Pourhassan, International Journal of Modern Physics D Vol. 22, No. 9 (2013) 1350061 [arXiv:1301.2788 [gr-qc]]

[15] A.R. Amani and B. Pourhassan, International Journal of Theoretical Physics 52 (2013) 1309

[16] H. Saadat and B. Pourhassan, Astrophysics and Space Science 344 (2013) 237

[17] H. Saadat and B. Pourhassan, Astrophysics and Space Science 343 (2013) 783

[18] H. Saadat, H. Farahani, International Journal of Theoretical Physics 52 (2013) 1160

[19] J. Sadeghi, H. Farahani, "Interaction between viscous varying modified cosmic Chaplygin gas and Tachyonic fluid", Astrophysics and space science journal (2013), [arXiv:1304.6987 [gr-qc]] 
[20] H. Saadat, B. Pourhassan, "Effect of Varying Bulk Viscosity on Generalized Chaplygin Gas", [arXiv:1305.6054 [gr-qc]]

[21] M.R. Setare, Chin. Phys. Lett. 26 (2009) 029501

[22] S.D.H. Hsu, Phys. Lett. B 594 (2004) 13

[23] M.R. Setare, E. N. Saridakis, Phys. Lett. B671 (2009) 331

[24] M. Jamil, E.N. Saridakis, M.R. Setare, Phys. Lett. B679 (2009) 172

[25] J. Lu, E.N. Saridakis, M.R. Setare, L. Xu, JCAP 1003 (2010) 031

[26] M.R. Setare, M. Jamil, JCAP 1002 (2010) 010

[27] M.R. Setare, M. Jamil, Phys. Lett. B690 (2010) 1

[28] Kh. Saaidi, A. Aghamohammadi, M.R. Setare, Astrophys. Space Sci. 332 (2011) 503

[29] M.R. Setare, Elias C. Vagenas, Phys. Lett. B666 (2008) 111

[30] S. Pan, S. Chakraborty, "Interacting Holographic Dark Energy: Scalar Field Models", [arXiv:1210.0396 [gr-qc]]

[31] A. Khodam-Mohammadi, M. Malekjani, Commun. Theor. Phys. 55 (2011) 942

[32] K. Karami, M.S. Khaledian, M. Jamil, Phys. Scr. 83 (2011) 025901

[33] F. Adabi, K. Karami, F. Felegary, Z. Azarmi, Res. Astron. Astrophys. 12 (2012) 26

[34] B. Guberina, R. Horvat, H. Nikolic, JCAP 01 (2007) 012

[35] A. Pourdarvish, J. Sadeghi, H. Farahani, and B.Pourhassan, International Journal of Theoretical Physics, DOI: 10.1007/s10773-013-1658-4

[36] J.D. Bekenstein, Phys. Rev. D 9 (1974) 3292

[37] J. Sadeghi, B. Pourhassan and F. Pourasadollah, Physics Letters B 720 (2013) 244 [arXiv:1209.1874 [hep-th]]

[38] J.D. Bekenstein, Phys. Rev. D 7 (1973) 2333

[39] J. Sadeghi, K. Jafarzade, and B. Pourhassan, "Thermodynamical Quantities of HoravaLifshitz Black Hole", International Journal of Theoretical Physics 51 (2012) 3891

[40] J.D. Bekenstein, Phys. Rev. D 23 (1981) 287

[41] J. Sadeghi, M. R Setare and B. Pourhassan, Acta Physica Polonica B 40 (2) (2009) 251 [arXiv:0707.0420 [hep-th]] 
[42] J.D. Bekenstein, Phys. Rev. D 49 (1994) 1912

[43] J. Sadeghi, M.R. Setare, B. Pourhassan, Eur. Phys. J. C 53 (2008) 95

[44] S.W. Hawking, Commun. Math. Phys. 43 (1975) 199

[45] J. Sadeghi, B. Pourhassan, A. Asadi, "Thermodynamics of string black hole with hyperscaling violation", [arXiv:1209.1235 [hep-th]]

[46] S.W. Hawking, Phys. Rev. D 13 (1976) 191

[47] H. Saadat, International Journal of Theoretical Physics 52 (2013) 1027

[48] N. Radicella, D. Pavon, J. Cosmol. Astropart. Phys. 10 (2010) 005

[49] R. Banerjee, B.R. Majhi, Phys. Lett. B 662 (2008) 62

[50] R. Banerjee, B.R. Majhi, JHEP 06 (2008) 095

[51] J. Zhang, Phys. Lett. B 668 (2008) 353

[52] H. Wei, Commun. Theor. Phys. 52 (2009) 743

[53] L.N. Granda, A. Oliveros, Phys. Lett. B669 (2008) 275

[54] L.N. Granda, A. Oliveros, Phys. Lett. B671 (2009) 199 